Écrire

l'histoire

\section{Écrire l'histoire}

Histoire, Littérature, Esthétique

9| 2012

Mensonges (1)

\title{
La cruauté, le rire, le sarcasme, l'histoire
}

À propos de Heinrich von Kleist et Heiner Müller

Jean Jourdheuil

\section{(2) OpenEdition}

Journals

Édition électronique

URL : http://journals.openedition.org/elh/269

DOI : 10.4000/elh.269

ISSN : 2492-7457

Éditeur

CNRS Éditions

\section{Édition imprimée}

Date de publication : 10 juin 2012

Pagination : 155-164

ISBN : 978-2-35698-046-5

ISSN : 1967-7499

Référence électronique

Jean Jourdheuil, « La cruauté, le rire, le sarcasme, l'histoire », Écrire l'histoire [En ligne], 9 | 2012, mis en ligne le 10 juin 2015, consulté le 23 septembre 2020. URL : http://journals.openedition.org/elh/269 ; DOI : https://doi.org/10.4000/elh.269 


\section{La cruauté, le rire, le sarcasme, 1'histoire $\grave{A}$ propos de Heinrich von Kleist et Heiner Müller}

Comment empêcher que le comédien ne fasse qu'un avec la scène, comme le fonctionnaire avec son bureau. Heiner Müller

\section{René Allio et le « film historique "}

Certains auteurs dans l'après-Brecht s'attacheront à donner une « représentation » de l'histoire, d'une histoire, au risque de la sacraliser: Peter Hacks avec La Bataille de Lobositz, les auteurs de «théâtre documentaire », Rolf Hochhuth (Le Vicaire), Peter Weiss (L'Instruction), Heinar Kipphardt (En cause 7. Robert Oppenheimer). Ainsi en va-t-il aussi de l'histoire (et de la géographie) dans les quatre films de René Allio auxquels j’ai collaboré: Les Camisards (1970) dans les Cévennes, Moi, Pierre Rivière... (1976) dans le bocage normand, et Un médecin des Lumières (1988) dans le Bourbonnais. Ces paysages étaient les dépositaires métaphoriques de l'unité de lieu. Dans le quatrième, Transit (1990), d'après le roman mi-témoignage mi-fiction dans lequel Anna Seghers évoque le séjour et l'attente des émigrés allemands en quête d'un visa et d'un bateau (pour les États-Unis ou le Mexique), l'unité de lieu, c'était Marseille, par excellence le site psychologique de René Allio dans plusieurs autres films: La Vieille Dame indigne, Retour à Marseille, L'Heure exquise. Le cinéma de René Allio s’inscrit dans une tradition française (dont l'unité de lieu est un indice) où le « film historique » n'est plus un « film à costumes », renonce au «planétarium » brechtien, ne se contente plus de «représenter», devient « concret et évocateur », est habité par l'inquiétude du vrai. Sa caméra dans Les Camisards tentait d'appréhender de manière encore brechtienne un groupe, une population, un collectif : les paysans et artisans protestants des Cévennes confrontés aux troupes royales. Dans les films ultérieurs $U n$ médecin des Lumières et Transit, elle s'approchait avec

Jean Jourdheuil, Université Paris Ouest La Défense, UFR de Philosophie Information-Communication Langage Littérature Arts du Spectacle (PHILLIA). 
tact tantôt d'un personnage, tantôt d'un autre, elle s'intéressait aux anonymes, ceux qui ne sont pas illustres et que Michel Foucault appelait les « hommes infâmes ${ }^{1}$ ».

\section{Brecht et la représentation de l'histoire}

Brecht ne fut pas l'auteur d'un «théâtre historique » qui aurait eu vocation à supplanter le «théâtre historique» des romantiques. À quelques exceptions près cependant: Les fours de la Commune, pièce écrite en 1949, à l'époque où lui et les siens s'installaient en RDA, à vrai dire, une adaptation réalisée à partir de la pièce de Nordhal Grieg, Nederlaget (La Défaite), qui avait été traduite par Margarete Steffin à la fin des années trente - cette pièce peut à la rigueur être considérée comme une « pièce historique ». C'est aussi l'une des pièces les plus conventionnelles de Brecht. Sa création, envisagée au Deutsches Theater, était censée permettrel'établissement de relations plutôt bonnes, c'est-à-dire modérément conflictuelles, avec les autorités de la toute nouvelle République Démocratique Allemande. Autre exception: Les Visions de Simone Machard, pièce écrite (en 19421943) en collaboration avec l'auteur de « romans historiques» Lion Feuchtwanger, conçue par Brecht et Feuchtwanger non seulement comme une pièce mais aussi comme un possible projet cinématographique et qui peut, à ce titre, être comparée au «script» de Brecht de 1942 qui a servi de base au scénario d'un film de Fritz Lang, Les bourreaux meurent aussi.

Malgré son intérêt singulier pour la pensée marxiste, Brecht n'a pas manifesté une grande attirance pour un «théâtre historique ». Il préférait le genre de la "pièce parabole »: Mère Courage faisant des affaires comme cantinière, suivant les armées pendant la guerre de Trente Ans et perdant successivement ses fils et sa fille ${ }^{2}$. La représentation de l'histoire n'y est pas niée mais, en quelque sorte, modélisée, imaginée et réfléchie comme un cas de figure; Brecht, auteur et metteur en scène, réfléchit et pense à haute voix, de manière certes figurative mais sans prétendre représenter l'histoire. Le caractère lacunaire de la représentation est ce qui laisse (le cas échéant et pour peu que le spectateur y soit enclin) un espace à la réflexion et à l'imagination du spectateur. Celui-ci, en effet, peut appréhender ce qui lui est proposé de bien des manières (le cas échéant en prenant le contrepied de la philosophie brechtienne de la représentation). La parole de Brecht, bien qu'éprise de clarté, ne me paraît pas, à proprement parler, contraignante. Même dans son théâtre dit de la maturité (Galilée, Mère Courage, Puntila et son valet Matti, Le Cercle de craie caucasien, etc.), quelque chose persiste de sa philosophie et de son esthétique du « dispositif expérimental».

1. La collaboration d'Allio avec Michel Foucault, Jean-Pierre Peter et Arlette Farge fut le lieu de ce « tournant ».

2. Voir l'analyse de Roland Barthes dans Écrits sur le théâtre, Éd. du Seuil, 2002, p. 272-292. 


\section{Le Lehrstück, le rire, la cruauté}

L'expression «dispositif expérimental» est utilisée par Walter Benjamin à propos des textes de Kafka et des "Lehrstücke" de Brecht (pièces pour apprendre, improprement appelées en français pièces didactiques), vaste chantier laissé inachevé auquel Brecht travailla à la fin des années vingt, au début des années trente et encore un peu dans ses premières années d'exil. Les deux dernières pièces didactiques, moins radicalement expérimentales, renouent avec l'idée de représentation, il est vrai à la manière du « théâtre chinois »: L'Exception et la Règle, pièce écrite de 1930 à 1934 et modifiée en 1937, Les Horaces et les Curiaces, écrite en 1934-1935. Le Lehrstück L'Importance d'être d'accord, souvent désigné comme Lehrstück de Baden-Baden (1929), comporte une scène de clowns beckettienne avant la lettre qui est censée montrer «comment des hommes viennent en aide à un homme»; le corps de Monsieur Schmitt est littéralement mis en pièces, démonté: les pieds puis les jambes sont amputés (à la scie), les oreilles puis la tête sont dévissées, les bras à leur tour sciés, et toutes ces parties du corps sont posées sur les genoux de Monsieur Schmitt - conclusion de cette scène de clowns: «l'homme n'est pas une aide pour l'homme ».
Les Lehrstücke sont le «théâtre de la cruauté » de Bertolt Brecht. Quelques spectateurs se sont évanouis alors que les acteurs sciaient vraiment, matériellement, les bras et jambes (de bois) de Monsieur Schmitt. Brecht, probablement, riait. Comme les auditeurs et Kafka lui-même lors de la lecture du Procès par l'auteur, et aussi, un siècle plus tôt, les auditeurs et Kleist lui-même lors de la lecture de La Famille Schroffenstein par son auteur ${ }^{3}$. La cruauté fait rire. Elle fait rire notamment celui qui s'imagine qu'il pourrait bien en être la victime.

Écrivant cela, je me souviens de la façon qu'avait Heiner Müller de lire Kafka:

Kafka continue Dostö̈evski dans un autre contexte. Kafka, en tant que juif germanophone à Prague, était a priori exclu. Son principal travail consistait à se sélectionner lui-même. Il s'est, par son écriture, transformé lui-même en prisonnier de camp de concentration. De là son réalisme. La dernière déclaration de Mielke ${ }^{4}$ à la Chambre du peuple fut: «Et pourtant je vous aime tous. » Kafka écrit dans Le Verdict: "Chers parents, pourtant je vous aimais. » C'est la dernière phrase du condamné avant qu'il se jette du haut du pont. Mielke ne connaissait certainement pas Kafka mais Kafka connaissait Mielke. ${ }^{5}$

3. La pièce lue était la première version de La Famille Schroffenstein, intitulée La Famille Ghonorez.

4. Ministre de la Sécurité d'État, en d'autres termes chef de la Stasi, de 1957 à 1989.

5. Heiner Müller, «Penser est fondamentalement coupable », dans Fautes d'impression, textes et entretiens choisis par Jean Jourdheuil, L'Arche, 1991, p. 196-197.

Jean Jourdheuil, « La cruauté, le rire, le sarcasme, l'histoire. À propos de Heinrich von Kleist et Heiner Müller » 


\section{L'ours et l'intellectuel selon Heiner Müller}

La première scène de la pièce intitulée Vie de Gundling Frédéric de Prusse Sommeil rêve cri de Lessing nous présente une soirée royale à Potsdam, jardin, nuit, bière et tabac; Friedrich Wilhelm, le Roi-Sergent, avec ses officiers et le jeune Frédéric dont l'éducation royale reste à faire: le futur monarque éclairé Frédéric II n'est pas très viril, joue de la flûte, a quelques penchants pour l'homosexualité et apprécie exagérément les «tragédies françaises ». Le monarque et ses officiers s'amusent quant à eux de manière tout à fait virile aux dépens de l'intellectuel Jakob Paul, baron de Gundling, tenu d'offrir une tournée générale pour fêter chacune de ses innombrables promotions: président de l'Académie royale, conseiller à la Cour de cassation, maître des cérémonies, conseiller à la cour d'appel, etc. Il s'agit de nominations «pour rire ». L'intellectuel, à la cour de Friedrich Wilhelm, tient le rôle du bouffon. Lorsque enfin Gundling est ivre, les officiers font entrer sa fiancée, un ours aux pattes rognées, aux dents brisées. Poursuite autour de la table. L'ours enlace Gundling. Commentaire de Friedrich Wilhelm à l'intention de son fils :

Que ce soit un exemple pour lui de ce qu'il convient de penser des intellectuels. Et de l'art du gouvernement [...]. Au peuple, cette bête, lui rogner les pattes et lui briser les dents. L'intelligence, la tourner en ridicule, afin que la populace n'aille pas se faire des idées.

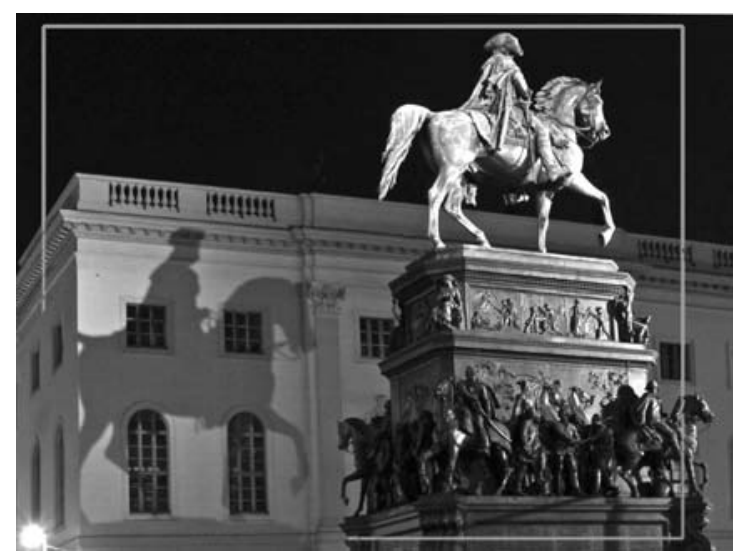

Statue équestre du roi Frédéric II de Prusse par Christian Daniel Rauch inaugurée en 1851. cliché: cdw (wrere.cdw-be.de)

C'était, selon Heiner Müller, la Prusse à l'aube du $\mathrm{XVIII}^{\mathrm{e}}$ siècle.

L'historien Jakob Paul baron de Gundling achèvera sa carrière terrestre dans un cercueil d'un genre particulier: un tonneau d'eau-de-vie ou de vin. Dans Berlin, aujourd'hui, une statue équestre de Frédéric de Prusse, le monarque éclairé, surplombe l'avenue Unter den Linden à proximité de l'université Humboldt. Transportée à Potsdam en 1951, lorsque la « république des ouvriers et des paysans » pensait pouvoir se passer des emblèmes de la monarchie prussienne, elle a retrouvé en 1980 «sa» place entre la Staatsbibliothek et l'université Humboldt. Sur le socle de cette statue, à l'étage inférieur, la galerie des hommes d'État, des architectes, des artistes, des intellectuels. Les intellectuels sont rassemblés sous le cul du cheval.

Cette pièce s'achève sur un triptyque intitulé Sommeil rêve cri de Lessing, trois scènes qui traitent, 
certes, du grand écrivain allemand d'origine saxonne Lessing, mais plus encore de Heiner Müller, Saxon lui aussi, sous le masque de Lessing. Acteur que l'on maquille (masque de Lessing). Il lit « Mon nom est Gotthold Ephraim Lessing. J'ai 47 ans. » L'âge de Heiner Müller lorsqu'il écrivait cette pièce. « [...] J'ai vu se lever une ère nouvelle après l'autre, chacune suintant de tous ses pores sang excréments sueur. L'histoire atteint la ligne d'arrivée sur des chevaux morts. » $\mathrm{Au}$ début des années soixante-dix, alors qu'il tentait avec d'autres (Peter Hacks, Helmut Baierl, Karl Mickel, Volker Braun) et en concurrence avec eux de prolonger la voie entrouverte par Bertolt Brecht de son vivant, vite colmatée et murée à sa mort, Heiner Müller fut accusé de «pessimisme historique ». «J'ai vu l'enfer des femmes d'en bas: la femme à la corde la femme aux veines ouvertes la femme à l'overdose SUR LES LÈVRES DE LA NEIGE la femme à la tête dans la cuisinière à gaz. » Ces quelques lignes qui s'ouvrent sur une citation de Rimbaud font allusion aux nombreuses tentatives de suicide d'Inge Müller, l'épouse de Heiner Müller morte en 1966.

Cette première séquence: Sommeil de Lessing, fait écho à la vie de Heiner Müller dans les années soixante après le suicide d'Inge Müller. La seconde : Rêve de Lessing, à son séjour aux ÉtatsUnis en 1975-1976, lorsqu'il fut «professeur invité » à l'université d'Austin au Texas ${ }^{6}$ : « DE LA PRUSSE DE FRÉDÉRIC DEUX MÉDAILLE D'OR AU PAS DE L'OIE MÉDAILLE D’ARGENT AU PASSAGE PAR LES VERGES LESSING VIENT EN AMÉRIQUE PAYS DE LA POMME DE TERRE QUI FERA LA GRANDEUR DE LA PRUSSE DANS UN CIMETIÈRE DE VOITURES DANS LE DAKOTA IL RENCONTRE LE DERNIER PRÉSIDENT DES ÉTATS-UNIS ». Ce dernier président des ÉtatsUnis n'est autre que Charles Manson, auteur avec sa family de la tuerie de Los Angeles où Sharon Tate fut assassinée (en 1969): Manson déclara à son procès que le président des ÉtatsUnis avait commis plus de crimes que lui. Troisième séquence: Cri de Lessing: PROJECTION : apothéose spartacus un Fragment. Cette projection énigmatique fait allusion au fait que Lessing avait eu le projet d'écrire une pièce consacrée à Spartacus, pièce demeurée à l'état de fragment (quelques pages). La didascalie décrit une pantomime dont voici les dernières lignes: Les garçons de café, à présent avec casques de protection, essayent à Lessing un buste de Lessing qui recouvre sa tête et ses épaules. Lessing, à genoux, fait de vaines tentatives pour se libérer du buste. On entend son cri sourd sous le bronze. Applaudissements des garçons de café, machinistes (spectateurs). La chute dans la gloire n'advient pas sans quelques contreparties.

De Gundling à Lessing, en passant par Frédéric II, roi et intellectuel, et par Heinrich von

6. Il était alors membre de la «dramaturgie » du Berliner Ensemble et son invitation aux États-Unis fut, à ce titre, soutenue par la directrice du Berliner Ensemble, Ruth Berghaus.

Jean Jourdheuil, « La cruauté, le rire, le sarcasme, l'histoire. À propos de Heinrich von Kleist et Heiner Müller » 
Kleist dans une scène de pantomime, une scène muette: Heinrich von Kleist joue Michael Kohlhaas Kleist est, probablement pour cette raison, le seul à n'avoir pas été signalé dans le titre à tiroirs de la pièce -, c'est à une déclinaison de la figure de l'intellectuel en Prusse que nous sommes conviés. L'intellectuel ou celui qui doit apprendre à rire de son propre malheur et le cas échéant à chanter lorsqu'il est dans la merde jusqu'au cou. Seuls y parviennent ceux qui ont une âme de clown. Pour les autres, les anonymes voués à la corvée, l'histoire ne prête pas à rire.

\section{L'ours et la marionnette selon Heinrich von Kleist}

Dans son essai Sur le théâtre de marionnettes (1810), Kleist expose en trois temps et en prenant trois exemples une conception de la grâce qui est aussi un impossible programme esthétique. Il y est question d'une marionnette de facto gracieuse dans la mesure où elle coïncide avec son centre de gravité : l'appareillage de fils équilibre la force de la pesanteur - dès lors que le centre de gravité se déplace la marionnette danse et aucun danseur ne peut espérer rivaliser avec sa grâce, il lui faut s'accommoder de «l'inertie de la matière ».

Il est question ensuite d'un jeune homme, un éphèbe dit le texte, dont les mouvements cessèrent d'être gracieux lorsqu'il se mit en tête de les étudier en s'aidant d'un miroir afin de les fixer et de les reproduire.
Et enfin, il est question d'un ours capable de tenir en échec le meilleur escrimeur :

[...] l'ours se tenait debout sur ses pattes de derrière, adossé à un pieux où il était attaché, la patte droite levée, prête à frapper, et il me regardait droit dans les yeux: c'était sa position d'escrime. Je ne savais pas si je rêvais en me retrouvant face à un tel adversaire. [...] Revenu un peu de mon étonnement, je poussai une botte; l'ours fit un bref mouvement de la patte et para le coup. Je tentai de l'abuser par des feintes, l'ours ne bougea pas. Je fondis à nouveau sur lui avec une si soudaine agilité que j'aurais infailliblement touché une poitrine humaine: l'ours fit un bref mouvement de la patte et para le coup. [...] Non seulement l'ours, pareil au meilleur escrimeur du monde, parait toutes mes attaques mais il ne réagissait à aucune de mes feintes (ce que ne fait aucun escrimeur au monde): le regard fixé dans le mien, comme s'il pouvait lire dans mon âme. Il était là, debout, la patte levée, prête à frapper; et quand mes attaques n'étaient pas portées pour de vrai, il ne bougeait pas.

Le juriste de la «droite conservatrice» allemande Carl Schmitt, dans un texte intitulé Deux tombeaux, où il met étrangement en parallèle les suicides d'officiers de la Wehrmacht à la fin de la Seconde Guerre mondiale et le suicide de Kleist en 1811, voyait dans cet essai, et dans cette figure de l'ours, un indice de la position géopolitique clivée de Heinrich von Kleist: entre l'Est asiatique et l'Occident. Kleist était aussi un génie que la fascination de la mort n'avait pas épargné. Son suicide, avec Henriette Vogel, au bord du Wannsee, cite un tableau qu'il avait vu à Châlons-sur-Marne en 1808. Même à l'instant 

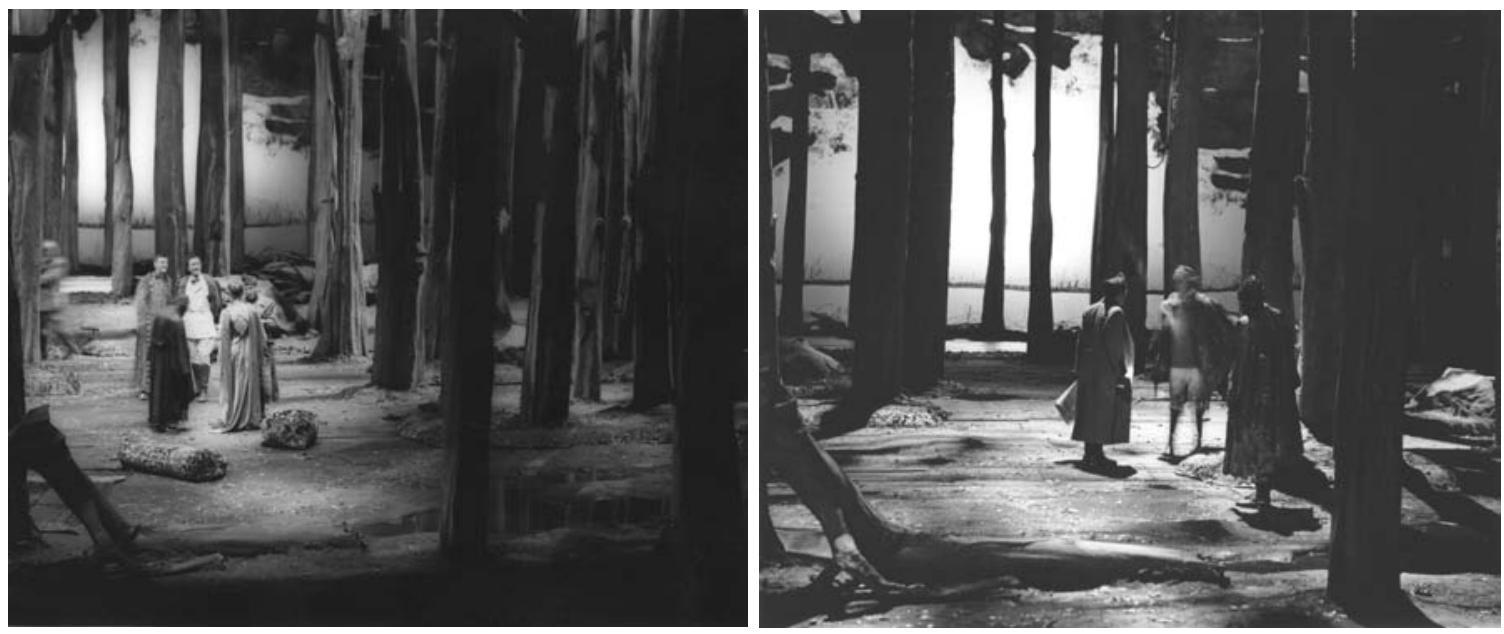

La Bataille d'Arminius de Heinrich von Kleist (décor, Gilles Aillaud; mise en scène Jean Fourdheuil, en mars 1995 au Théatre Nanterre Amandiers). Cliché: Thierry Gründler

de la mort, il ne résistait pas au plaisir de la citation ${ }^{7}$.

\section{L'ours, la barbarie et la géopolitique chez Kleist}

Descendant d'une ancienne famille prussienne, Kleist naquit à Francfort-sur-l'Oder, ville située actuellement à la frontière entre l'Allemagne et la Pologne; de son temps, la Prusse s'étendait au moins jusqu'à Poznan. Un de ses petits-neveux, un siècle plus tard, Heinrich Ewald von Kleist, presque un homonyme dont les deux prénoms disent la vénération portée aux deux poètes de la lignée, participa à la conspiration contre Hitler. Il devait se jeter au cou du Führer lors d'une présentation des nouveaux uniformes destinés aux soldats du front de l'Est. Les Anglais ayant bombardé l'usine qui les fabriquait, la visite du Führer et le projet d'attentat furent annulés. Heinrich Ewald von Kleist fut l'un des rares survivants de la conspiration conduite par Stauffenberg. Lui et Manfred Rommel, la post-Wehrmacht parvenue à un âge canonique, accompagnèrent Helmut Kohl à Paris en juillet 1989 lors de la cérémonie dite du Bicentenaire de la Déclaration des droits de l'homme, un an à peine avant la réunification de l'Allemagne. Un autre Kleist, qui lui non plus n'était pas nazi, Ludwig Paul Ewald von Kleist, l'un des généraux de premier plan de la Seconde Guerre mondiale, un virtuose de la «bataille de

7. Cf. la biographie de Kleist par Peter Michalzik, Kleist. Dichter, Krieger, Seelensucher, Berlin, Propyläen, 2011. 
chars » avec Guderian et Rommel, commanda le Panzergruppe Kleist puis la 1. Panzer-Armee. Il adressa un jour à l'état-major, en guise de rapport, un pentamètre iambique: "Vor mir kein Feind, hinter mir kein Nachschub ", " Devant moi nul ennemi, derrière nul renfort » - c'était à l'époque de l'offensive en direction du Caucase et des champs pétrolifères, avant la défaite de Stalingrad. Après la guerre, Ludwig Paul Ewald von Kleist, arrêté par les Américains en Bavière, fut livré aux Yougoslaves, qui le condamnèrent pour «crime de guerre » avant de le livrer aux Soviétiques. Il mourut en 1954, en captivité, au camp de Vladimir en URSS. Il y a dans cette lignée des Kleist toute une géologie de la chose militaire continentale.

L'unité de l'Allemagne, la fondation de l'Empire allemand négociée avec obstination par Bismarck, adviendra tardivement, après la guerre de 1870. Elle sera proclamée en 1871 à Versailles. Mais le sentiment national s'était développé dans les principautés allemandes dès le début du siècle, lors de l'occupation napoléonienne à la suite de la défaite de la Prusse à Iéna en 1806. Le poète Heinrich von Kleist haïssait Napoléon et voulait l'unité de l'Allemagne. Sa pièce la Hermannsschlacht, Bataille d'Hermann ou d'Arminius, qui à première vue, lorsqu'on se contente d'une lecture superficielle, représente l'arrivée des légions de Varus au pays des Chérusques et leur extermination dans la forêt de Teutoburg, est à double fond. Elle joue sur un double registre historique. Sous le masque des Romains, elle traite de l'occupation napoléonienne; elle est, au théâtre, un appel à la lutte, au soulèvement contre l'occupant français. La figure d'Hermann dans la pièce de Kleist emprunte un certain nombre de traits et de conduites de double jeu à l'égard de l'occupant au Freiherr vom Stein qui, dans l'entourage du roi de Prusse (lequel avait fait acte d'allégeance), tentait avec quelques autres (Scharnhorst, Gneisenau) d'organiser contre les Français la résistance et une guerre de partisans, le Kleinkrieg. On a pu dire de la pièce de Kleist qu'elle était par une sorte d'anticipation nietzschéenne Die Geburt des Partisanen aus dem Geist der Poesie ${ }^{8}$ (La naissance du partisan à partir de l'esprit de la poésie).

Je retiens de cette pièce, dont l'actualité persistante excède la notion de « drame historique », comme si Kleist s'était attaché avec âpreté et non sans sarcasme à dépasser le «politiquement correct» du «drame historique » selon Schiller, deux moments, deux épisodes qui ouvrent l'espace de la barbarie, de la cruauté, de l'histoire.

Tout d'abord, à l'acte IV, les scènes 4, 5 et 6, où une jeune fille violée par des soldats romains est exécutée par son propre père (et ses cousins) et dont le corps ensuite, par décision d'Hermann, est

8. Titre d'un essai de Wolf Kittler (Fribourg-en-Brisgau, Rombach) qui porte en sous-titre : « Heinrich von Kleist et la stratégie des guerres de libération » [Heinrich von Kleist und die Strategie der Befreiungskriege]. 
découpé en quinze morceaux envoyés aux quinze tribus de Germanie pour les inciter à se soulever et à sceller de ce fait leur unité. Cet épisode macabre et cruel est une citation de la Bible (Le crime de Gibéa et la guerre contre Benjamin) que Kleist a probablement méditée dans la version qu'en a donnée Jean-Jacques Rousseau dans Le Lévite d'Ephrä̈m.

L'autre épisode concerne Thusnelda, l'épouse d'Hermann. Elle s'était montrée sensible aux compliments et aux avances que lui prodiguait Ventidius, le légat de Rome. Elle ira même jusqu'à demander sa grâce lorsque Arminius l'informera, à la dernière minute, de la ruse, du piège imminent par lequel il compte exterminer tous les Romains auxquels jusqu'ici il a fait bonne figure pour mieux endormir leur vigilance.

Ventidius, peu auparavant, avait coupé une boucle blonde de la chevelure de Thusnelda. Hermann informe alors Thusnelda qu'un courrier de Ventidius adressé à l'Impératrice de Rome avait été intercepté et qu'il contenait cette boucle que Ventidius envoyait à son impératrice comme un échantillon d'une perruque blonde dont elle, l'Impératrice aux cheveux noirs, pourrait bientôt se parer. La transformation qui s'opère en Thusnelda - souvent appelée Thusschen par Hermann - est radicale. Elle était séduite par les « modes françaises », elle devient furieuse, cruelle, bestiale, elle n'a plus d'objectif que la vengeance. À l'instar d'autres figures kleistiennes, elle passe sans transition du registre divin, de l'aspiration au divin, au registre animal.

Le soir même, alors que son mari vient de partir pour Teutoburg, elle donne rendez-vous à Ventidius dans l'idyllique petit bois de chênes, comme il le lui avait demandé.

Le soir venu (acte V, scène 17), sa servante reculant devant la monstruosité imminente, Thusnelda prend le rôle de la servante défaillante et, dans l'obscurité, introduit Ventidius dans la cage où l'ourse noire de Chérusquie « récemment capturée par Hermann » et « n’ayant rien mangé depuis douze heures » a été, par Thusnelda, substituée à elle-même, à la princesse des Chérusques prête à succomber aux charmes du Romain. Désiré il y a peu, désormais déchiré ${ }^{9}$, le légat de Rome succombera non sans découvrir ce qu'il convient de penser du « devenir animal » d'une princesse germanique. La cérémonie s'achève sur l'évanouissement de la princesse. Cette scène est une variante rustique de la mort d'Achille et de l'évanouissement de Penthésilée dans la pièce du même nom.

Ventidius, avec épouvante

Zeus, père des dieux et des hommes!

Quel est ce monstre de l'enfer?

Thusnelda, à travers la grille

Qu'y a-t-il, Ventidius? Qu'est-ce qui t'effraie?

9. C'est ainsi que Julien Gracq traduit Küsse Bisse, «baisers morsures », dans Penthésilée: Küsse Bisse es reimt sich, « Désirer... Déchirer... cela rime. »

Jean Jourdheuil, « La cruauté, le rire, le sarcasme, l'histoire. À propos de Heinrich von Kleist et Heiner Müller » 
Ventidius

L'ourse velue et noire de Chérusquie

Se dresse à côté de moi, toutes griffes dehors!

\section{Thusnelda}

L'ourse de Chérusquie?

[...]

C'est Thusnelda, tu as deviné, la princesse,

Sur sa tête récemment tu as dérobé

Une boucle soyeuse, l'échantillon pour Livie!

Un moment si favorable, ne le laisse pas échapper,

Cajole-la et rase-lui toute la tête!

Ventidius

Zeus, père des dieux et des hommes,

Elle se redresse, tout est fini pour moi!

$[\ldots]$

\section{THUSNeLDA, à travers la grille}

$\mathrm{Ah}$, comme ces poils, très cher, noirs et rêches,

Embelliront Livie, ton impératrice,

Quand ils retomberont sur sa nuque!

Gouverneur de Chérusquie je te salue!

Tu mérites bien cela, fidèle valet,

En récompense de tes bons services!

Ventidius

Zeus, père des dieux et des hommes,

Elle plante ses griffes dans ma tendre poitrine!

\section{Thusnelda}

Thusnelda? Allons donc!

$[\ldots]$

Ventidius, avec douleur

Malheur! Malheur!

$[\ldots]$

Elle te résiste?

$[\ldots]$

Ventidius
Ah! Miséricorde! Malheur! Ô Thusnelda!
ThusNELDA
Dis-lui que tu l'aimes, Ventidius,
Elle sera docile et t'offrira ses boucles.
Elle jette la clef et s'évanouit. ${ }^{10}$

Ces deux scènes, celle de la jeune fille violée et dépecée et celle du Romain dévoré par l'ourse, dessinent la figure du « chaudron de sorcières » où s'engendra le nationalisme allemand. Si le Prince de Homburg est le Hamlet de Heinrich von Kleist, La Bataille d'Arminius est son Macbeth entre sorcières et forêt, la version allemande de la «tragédie de la vengeance » selon les élisabéthains.

10. La Bataille d'Arminius, trad. de Jean-Louis Besson et Jean Jourdheuil, Éditions théâtrales, 1995, p. 108-110. 